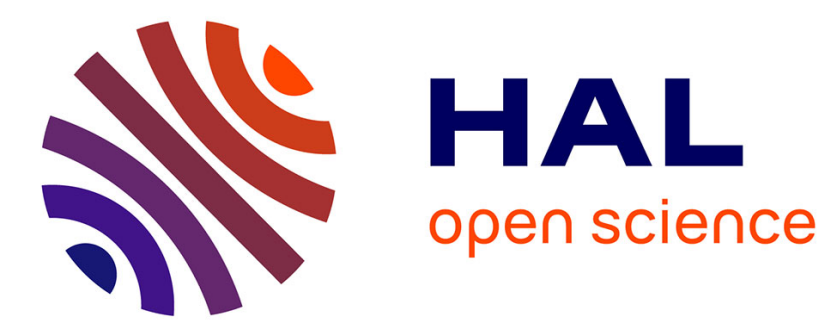

\title{
Minimizing Energy and Link Utilization in ISP Backbone Networks with multi-path Routing: A Bi-level Approach
}

Ikram Bouras, Rosa Figueiredo, Michael Poss, Fen Zhou

\section{- To cite this version:}

Ikram Bouras, Rosa Figueiredo, Michael Poss, Fen Zhou. Minimizing Energy and Link Utilization in ISP Backbone Networks with multi-path Routing: A Bi-level Approach. Optimization Letters, 2020, 14 (1), pp.209-227. 10.1007/s11590-019-01505-x . hal-02325913

\section{HAL Id: hal-02325913 \\ https://hal.science/hal-02325913}

Submitted on 22 Oct 2019

HAL is a multi-disciplinary open access archive for the deposit and dissemination of scientific research documents, whether they are published or not. The documents may come from teaching and research institutions in France or abroad, or from public or private research centers.
L'archive ouverte pluridisciplinaire HAL, est destinée au dépôt et à la diffusion de documents scientifiques de niveau recherche, publiés ou non, émanant des établissements d'enseignement et de recherche français ou étrangers, des laboratoires publics ou privés. 


\title{
Minimizing Energy and Link Utilization in ISP Backbone Networks with multi-path Routing
}

\author{
A Bi-level Approach
}

\author{
Ikram Bouras - Rosa Figueiredo • \\ Michael Poss · Fen Zhou
}

the date of receipt and acceptance should be inserted later

\begin{abstract}
In recent years, green networking has attracted a lot of attention from device manufacturers and Internet Service Providers (ISP) to reduce energy consumption. In the literature, energy-aware traffic engineering problem is proposed to minimize the total energy consumption by switching off unused network devices (routers and links) while guaranteeing full network connectivity.

In this work, we are interested in the problem of energy-aware Traffic Engineering while using multi-path routing (ETE-MPR) to minimize link capacity utilization in ISP backbone networks. To this end, we propose a bi-level optimization model where the upper level represents the energy management function, and the lower level refers to the deployed multi-path routing protocol. Then, we reformulate it as a one-level MILP replacing the second level problem by different sets of optimality conditions. We further use these formulations to solve the problem with classical branch-and-bound, cutting plane, and branchand-cut algorithms. The computational experiments are performed on real instances to compare the proposed algorithms and to evaluate the efficiency of our model against the existing single-path and multi-objective approaches.
\end{abstract}

\author{
I. Bouras \\ LIRMM, University of Montpellier, 161 rue Ada, 34095 Montpellier Cedex 5, France \\ E-mail: ikram.bouras@lirmm.fr \\ R. Figueiredo \\ CERI-LIA, University of Avignon, 84911 Avignon, France \\ E-mail: rosa.figueiredo@univ-avignon.fr \\ M. Poss \\ LIRMM, University of Montpellier, 161 rue Ada, 34095 Montpellier Cedex 5, France \\ E-mail: michael.poss@lirmm.fr \\ F. Zhou \\ CERI-LIA, University of Avignon, 84911 Avignon, France \\ E-mail: fen.zhou@univ-avignon.fr
}


Keywords Energy-aware engineering, Bi-level programming, Multi-path, Cutting plane, Branch-and-Cut.

\section{Introduction}

As we are entering the era of Internet of Everything, the number of devices connected to the IP networks will increase by one and a half times the global population ( 8 billion) by 2022, says the latest Cisco Visual Networking Index (VNI) 13. Consequently, the monthly global IP traffic will be 77 exabytes by 2022 , and the annual traffic will reach almost one zettabyte [13. With the tremendous growth of Internet traffic, the network energy consumption is inherently growing fast with a rate of $10 \%$ per year, which exceeded $350 \mathrm{TWh}$ and represented $1.8 \%$ of the worldwide electricity consumption in 2012 [18. It is even reported that communication networks will consume as much as $51 \%$ of the global electricity in the worst-case by 2030, if its energy efficiency is not improved enough [4. Thus, the problem of energy efficiency is becoming critical for communication networks nowadays.

To reduce energy consumption, green networking has attracted a lot of attention from device manufacturers and Internet Service Providers (ISP). To this end, many technologies and approaches have been proposed by the networking community to cut the carbon footprint of the ISP backbone networks 1, 3, 7, 10, 14, 16. Among them, one promising solution is the energy management of network elements including routers and communication links [1,3, 10, 12. In fact, network resources like processing power and memory are oversized in communication networks nowadays, which results in a low utilization of $30-40 \%$ in low traffic periods 14 . As a result, important energy savings can be obtained by switching into the sleeping mode the network elements which are not used for the data delivery during a certain period. Following this idea, authors in 10 tried to minimize the total energy consumption by switching off unused network devices while guaranteeing full network connectivity. In 3], the authors studied the energy-aware traffic engineering problem subject to elastic traffic and max-min fair bandwidth allocation. The work in 1 is focused on the energy minimization strategy that selectively switches off devices according to the traffic level. The authors of [12] proposed an OSPF-integrated routing strategy for QoS-aware energy saving in backbone networks.

However, all the work mentioned above only considered the single-path routing (using protocols like Routing Information Protocol (RIP) or Open Shortest Path First (OSPF) protocol) for the energy-aware traffic engineering [1,3, 10, 12, where only one path is used per communication request to deliver data from its source node to its destination. The single-path routing strategy may lead to a high blocking probability when some network links are congested during the period of high traffic load. In light of this, multi-path routing has been investigated [5, 9, 11, 25, 20, 21,26.

Recent works show that Internet-wide multi-path routing is feasible and very attractive: First, utilizing diverse paths through multi-path routing en- 
ables to reduce blocking probability by load balancing, which permits to improve both the utilization efficiency of network bandwidth and the transmission reliability. Second, a higher Quality of Service (QoS) and Quality of Experience (QoE) can be obtained [19,20,25]. Thus, many multi-path routing protocols and algorithms have been proposed, for instance, generalized destination-based multi-path routing [26, equal-cost multi-path routing [1] and minimum routing cost multi-path routing [21. In general, there are two main variants of multi-path routing, whose optimization goal is either to minimize the network congestion ratio through load balancing [5, 17, 23, 26, or to minimize the total routing cost of traffic flows on all paths [9,11,21].

In this paper, we deal with the problem of energy-aware traffic engineering while using multi-path routing to minimize link capacity utilization in ISP backbone networks. We suppose the network control plane disposes of several key functions like energy management, routing, and signaling. To achieve energy-efficient, we suppose that the energy management function and the deployed routing protocol work independently. On the one hand, to improve the energy efficiency, the energy management function of the ISP network tends to minimize the network energy consumption by putting on sleep unused network devices according to the actual traffic demands. On the other hand, the network operator should also assure that the deployed routing protocol is able to provide all the traffic demands in the link capacity constraint. We assume that the multi-path routing protocol minimizing total link capacity utilization (a simple version of total routing cost of all traffic flows on paths) [21,9,11] is used by the ISP network operator. However, the energy consumption and link capacity utilization cannot be optimized simultaneously, and an equilibrium should be achieved for the two control plane functions. Different from the literature, we propose a bi-level formulation for this green network optimization problem instead of the traditional single-level one 1 .

Our bi-level formulation is motivated by the following two reasons. First, from the point of view of the ISP network operator, it is difficult to tune the weights of two network resources (i.e., energy consumption and link capacity utilization) so as to find a good trade-off. In contrast, our formulation enables to find an equilibrium between the two network resources utilization, which is transparent for the ISP network operator. Second, instead of developing new energy-aware routing protocols, we can take advantage of the existing work by deploying directly the proposed multi-path routing protocols in [21, 9,11, which minimize the total link capacity utilization. In our bi-level model, the upper level represents the energy management function aiming at cutting the total network energy consumption. The lower level refers to the deployed multi-path routing protocol, whose objective is to reduce the total link capacity utilization.

The novelties and contributions of this work can be summarized as follows:

- We study the problem of minimizing the network energy consumption while using multi-path routing to reduce link capacity utilization. To the best of our knowledge, it is the first time that this problem is investigated. 
- From the perspective of optimization techniques, we formulate the studied problem as a bi-level optimization for the first time. To solve it, the bi-level formulation is then transformed into a one-level optimization using KKT conditions, minimum flow inequalities or residual network optimality conditions. A cutting plane and branch-and-cut methods are further proposed to solve the problem to optimality.

The rest of this paper is organized as follows. The energy-aware traffic engineering problem is presented in Section 2 and its bi-level formulation is given in Section 3. Then, in the same section, we reformulate the problem as a one-level MILP formulation by using different optimality conditions. In Section 4, several algorithms based on the MILP formulations are proposed to solve the studied problem. To verify the proposed models and solutions, numerical simulations have been conducted in Section 5 . Finally, Section 6 concludes this paper.

\section{Problem definition and notation}

We consider an ISP backbone network (Figure 1) with slow dynamics, where the traffic demand is supposed to be relatively stable during a period of time. With the help of intelligent network management like Software Defined Networking (SDN), the control plane of the ISP network disposes of key functions like energy management and routing. This kind of network management permits us to change the state of network devices and engineer the traffic in a centralized way.

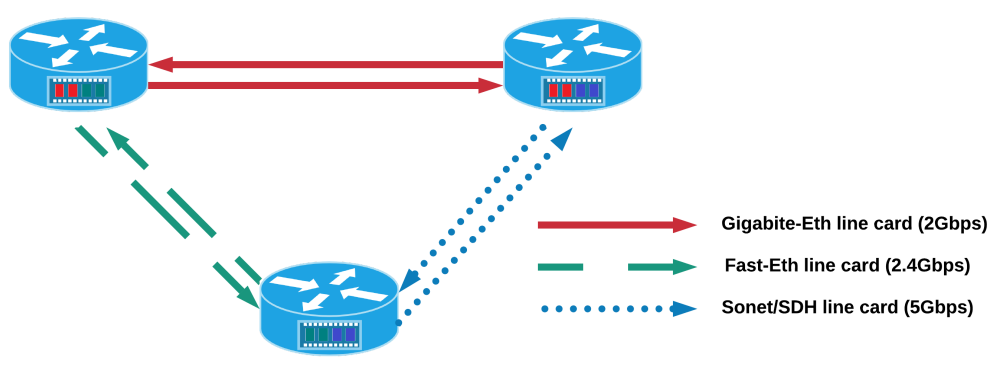

Fig. 1: ISP model architecture.

To correctly model the energy consumption of a router, we should get familiar with its architecture. We consider the widely used router architecture, which consists of a chassis and a set of line cards [1,3. The chassis is used to provide computation and switching functionalities. One example can be chassis Junifer M10i. Line cards are usually used to provide communication interfaces 
and network processing. To achieve power savings, the router elements (chassis and line cards) can be powered on and off by the control plane energy management function. Once activated, the chassis has a constant energy consumption independent from the amount of traffic passing through the router. It should be noted that the chassis can only be put into sleep when all of its line cards are OFF. To satisfy heterogeneous traffic, a router can be plugged with different kinds of line cards, which result in distinct energy efficiencies. For instance, we have line cards like Gigabit-Eth (2Gbps), Fast-Eth 12 ports (2.4Gbps), SONET /SDH OC-48c (5Gbps), among others, whose capacity and hourly power consumption are listed in [1,3. However, a communication link must be connected to the same type of line cards available on the two ending routers. Thus, each communication link is accordingly associated with a capacity and an energy-efficiency factor, which depends on the types of line cards used. For energy savings, only a subset of line cards are powered on to assure just enough bandwidth for the carried traffic while the rest will be put to sleep. For simplicity reasons, we thus assume the energy consumption on communication links is proportional to its carried traffic rate.

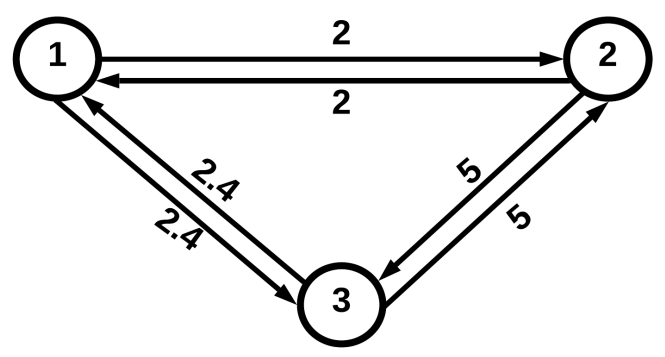

Fig. 2: The graph representation of the ISP network (Figure 1).

We consider the ISP backbone network modeled by the bi-directed graph $G(V, A)$ (Figure 2), where $V$ is the set of routers and $A$ represents the set of communication links. Since the backbone networks are bi-directed and for modelling the activation link constraints (Section 3), we define the set of (undirected) edges associate to $A$ as $E=\{\{i, j\}:(i, j) \in A$ or $(j, i) \in A\}$.

Let $C_{i j} \geq 0$ denotes the capacity of the link $(i, j) \in A$ defined by the line cards on routers $i$ and $j$, and $K$ be the set of traffic demands. Demand $k \in K$ must send a volume of $\phi^{k}$ from node $o(k)$ to node $d(k)$.We denote by $P_{i}$ the energy consumption of the chassis in router $i$, and we use $g_{i j}=g_{j i}$ to denote the energy efficiency of the line cards connecting the link $(i, j) \in A$.

The energy-aware traffic engineering with multi-path routing problem (ETEMPR) problem consists of selecting a set of routers (nodes) and links (edges) to be activated with minimum energy consumption. Assuming that each traffic demand uses a multi-path flow minimizing the total link capacity utilization. 


\section{Mathematical formulations}

In this section, we will introduce four different formulations for the ETE-MPR problem. We start by a bi-level integer programming model in Subsection 3.1 . then, the model will be transformed into three one-level MILP formulations (Subsections 3.2, 3.3 and 3.4). For comparison purposes, Subsection 3.5 will be devoted to present the unsplittable flow version of the energy-aware traffic problem.

\subsection{Bi-level formulation}

The problem is naturally cast as a bi-level optimization problem where the upper level represents the energy management function, and the lower level refers to the deployed multi-path routing protocol. For each $i \in V$ and $e(i, j)=$ $\{i, j\} \in E$, we introduce the binary variables $z_{i}$ and $y_{e}$ that represent the power status (ON/OFF) of router $i$ and link $e=\{i, j\}$, respectively. Furthermore, for each $k \in K$ and $(i, j) \in A$ let the continuous positive variable $x_{i j}^{k}$ be the flow on $(i, j)$ of demand $k$.

The problem belongs to the class of NP-hard problems and can be modeled as a bi-level mixed integer programming problem, as follows:

$$
\begin{aligned}
& \min \sum_{i \in V} P_{i} z_{i}+\sum_{(i, j) \in A} \sum_{k \in K} g_{i j} x_{i j}^{k} \\
& \text { s.t. } z_{i} \in\{0,1\}, \\
& \forall i \in V, \\
& y_{e} \geq z_{i}+z_{j}-1 \text {, } \\
& \forall e=\{i, j\} \in E \text {, } \\
& \forall e=\{i, j\} \in E \text {, } \\
& y_{e} \leq z_{i} \text {, } \\
& \forall e=\{i, j\} \in E \text {, } \\
& \min \sum_{k \in K} \sum_{(i, j) \in A} x_{i j}^{k} \\
& \text { s.t. } \sum_{(i, j) \in \delta^{+}(i)} x_{i j}^{k}-\sum_{(i, j) \in \delta^{-}(i)} x_{j i}^{k}=b_{i}^{k}, \quad \forall i \in V, \forall k \in K \text {, } \\
& \sum_{k \in K} x_{i j}^{k} \leq C_{i j} y_{e(i, j)}, \\
& \forall(i, j) \in A, \\
& x_{i j}^{k} \geq 0 \text {, } \\
& \forall(i, j) \in A, \forall k \in K \text {. }
\end{aligned}
$$

where:

$$
b_{i}^{k}=\left\{\begin{array}{lc}
\phi^{k}, & \text { if } i=o(k), \\
-\phi^{k}, & \text { if } i=d(k), \\
0, & \text { otherwise. }
\end{array}\right.
$$

The upper-level objective function (1a) aims to minimize the energy consumption of routers and links, while the objective of the second level problem is to 
minimize the total link capacity utilization $(1 \mathrm{f})$. Constraints $(1 \mathrm{c})-(1 \mathrm{e})$ ensure that a link $(i, j)$ is activated only if the two routers $i$ and $j$ are switched ON. Constraints $(1 \mathrm{~g})$ and $\sqrt{1 \mathrm{~h}}$ define the classical flow and capacity constraints, respectively. While 11 requires the variables $x_{i j}^{k}$ to be non-negative.

\subsection{One level formulation}

The problem can be reformulated as a one-level integer programming problem by replacing the second level problem by the associated optimality conditions. Following the classical reformulation used in [8,22, 15, among others, we can apply the fundamental theorem of duality and complementarity slackness conditions [6] to replace the follower problem:

$$
\begin{array}{llr}
\min & \sum_{i \in V} P_{i} z_{i}+\sum_{(i, j) \in A} \sum_{k \in K} g_{i j} x_{i j}^{k} & \\
\text { s.t. } & (1 \mathrm{~b})-(1 \mathrm{e}), & \\
& \sum_{(i, j) \in \delta^{+}(i)} x_{i j}^{k}-\sum_{(i, j) \in \delta^{-}(i)} x_{j i}^{k}=b_{i}^{k}, & \forall i \in V, \forall k \in K, \\
& \left(C_{i j} y_{e(i, j)}-\sum_{k \in K} x_{i j}^{k}\right) W_{i j}=0, & \forall(i, j) \in A, \forall k \in K, \\
& \left(1+\pi_{i}^{k}-\pi_{j}^{k}+W_{i j}\right) x_{i j}^{k}=0, & \forall(i, j) \in A, \forall k \in K, \\
& 1+\pi_{i}^{k}-\pi_{j}^{k}+W_{i j} \geq 0, & \forall(i, j) \in A, \forall k \in K, \\
& \sum_{k \in K} x_{i j}^{k} \leq C_{i j} y_{e(i, j)}, & \forall(i, j) \in A, \\
& W_{i j} \geq 0, & \forall(i, j) \in A, \\
& \pi_{i}^{k} \in \mathbb{R}, & \forall i \in V, \forall k \in K, \\
& x_{i j}^{k} \geq 0, & \forall(i, j) \in A, \forall k \in K .
\end{array}
$$

This formulation contains non-linear constraints $2 \mathrm{~b}$ and $2 \mathrm{c}$. We can linearize them using the big-M method by adding a new binary variable for each constraint. After this modification, we obtain a one-level mixed integer programming reformulation for (1), as follows: 


$$
\begin{array}{llr}
\min & \sum_{i \in V} P_{i} z_{i}+\sum_{(i, j) \in A} \sum_{k \in K} g_{i j} x_{i j}^{k} & \\
\text { s.t. } & 1 \mathrm{~b}]-(1 \mathrm{e}),(2 \mathrm{~d})-\sqrt{2 \mathrm{~h}}) & \\
& \sum_{(i, j) \in \delta^{+}(i)} x_{i j}^{k}-\sum_{(i, j) \in \delta^{-}(i)} x_{j i}^{k}=b_{i}^{k}, & \forall i \in V, \forall k \in K, \\
& C_{i j} y_{e e(i, j)}-\sum_{k \in K} x_{i j}^{k} \leq M \eta_{i j}, & \\
& M \eta_{i j}+W_{i j} \leq M, & \forall(i, j) \in A, \\
& 1+\pi_{i}^{k}-\pi_{j}^{k}+W_{i j} \leq M \mu_{i j}^{k}, & \forall(i, j) \in A, \forall k \in K, \\
& M \mu_{i j}^{k}+x_{i j}^{k} \leq M, & \forall(i, j) \in A, \forall k \in K, \\
& \eta_{i j} \in\{0,1\}, & \forall(i, j) \in A, \\
& \mu_{i j}^{k} \in\{0,1\}, & \forall(i, j) \in A, \forall k \in K .
\end{array}
$$

where $M$ is a precomputed large number. Unlike the other constraints, where the value of $M$ cannot be bounded, in constraint (3b) $M$ can be replaced by a lower bound $M=C_{i j}$ for each $(i, j) \in A$.

\subsection{BILP formulation based on flow constraints}

We propose an alternative one level formulation to the problem where the second level problem is replaced by a set of constraints eliminating unfeasible flows. Let us denote by $\mathcal{F}$ the set of all feasible flows defined as:

$$
\mathcal{F}=\{F=(x, y, z): 1 \mathrm{~b}-1 \mathrm{e}, 1 \mathrm{~g}-1 \mathrm{i})\}
$$

For each $F \in \mathcal{F}$, we also define $C(F)=\sum_{k \in K} \sum_{(i, j) \in A} x_{i j}^{k}$ as the total capacity link utilization of a flow $F$ and $G(F)=\left\{e \in E: y_{e}=1\right\}$ as the associated set of activated edges.

The flow $F=(x, y, z)$ is bi-level unfeasible if there exists a solution $F^{\prime}=$ $\left(x^{\prime}, y, z\right) \in \mathcal{F}$ such that:

$$
C\left(F^{\prime}\right)<C(F)
$$

Hence, the bi-level problem can be reformulated as: 


$$
\begin{array}{llr}
\min & \sum_{i \in V} P_{i} z_{i}+\sum_{(i, j) \in A} \sum_{k \in K} g_{i j} x_{i j}^{k} & \\
\text { s.t. } & 1 \mathrm{~b}-1 \mathrm{e} & \forall i \in V, \forall k \in K, \\
& \sum_{(i, j) \in \delta^{+}(i)} x_{i j}^{k}-\sum_{(i, j) \in \delta^{-}(i)} x_{j i}^{k}=b_{i}^{k}, & \forall F \in \mathcal{F}, \\
& \sum_{k \in K} \sum_{(i, j) \in A} x_{i j}^{k} \leq C(F)+\left(|G(F)|-\sum_{e \in G(F)} y_{e}\right) M, & \forall(i, j) \in A, \\
& \sum_{k \in K} x_{i j}^{k} \leq C_{i j} y_{e(i, j)}, & \forall(i, j) \in A, \forall k \in K . \\
& x_{i j}^{k} \geq 0, & \forall k,
\end{array}
$$

Constraint $4 \mathrm{~b}$ is in charge of eliminating all bi-level unfeasible flows $F$ on the set of activated links $\left\{e=\{i, j\} \in E: y_{e}=1\right\}$. Again, $M$ is a precomputed large number. The above formulation contains a polynomial number of variables and an exponential number of constraints.

\subsection{Residual network optimality conditions}

We can remark that the follower problem is a particular case of the minimum cost flow problem where the cost of each arc is equal to 1 . In this subsection, we propose to reformulate this problem by using a set of optimality conditions for the min-cost flow problems defined on the residual network.

We start by giving the definition of the residual network and the associated optimality conditions. Let $x^{k}=\left(x_{i j}^{k}\right)_{(i, j) \in A} \in \mathbb{R}^{|A|}$ be the flow between the origin-destination pair of demand $k \in K$. Notice that $x=\left(x^{k}\right)_{k_{1}, K} \in \mathbb{R}^{|A| \times|K|}$.

Definition 1 (Residual network) The residual network associated to $x^{k}$ is the graph denoted by $G^{k}(x)=\left(V, A^{k}(x)\right)$ and defined by the set of activated and unsaturated $\operatorname{arcs} A^{k}(x)$. This set of arcs is defined as the union of the two following sets:

$$
\begin{aligned}
& A^{k+}(x)=\left\{(i, j) \in A: 0<\sum_{k^{\prime} \in K} x_{i j}^{k^{\prime}}<C_{i j}, \quad x_{j i}^{k}=0\right\} \\
& A^{k-}(x)=\left\{(i, j) \in A: \quad x_{j i}^{k}>0\right\}
\end{aligned}
$$

Each arc of $G^{k}(x)$ is associated with a residual cost $c^{\prime k}$ defined as:

$$
c_{i j}^{\prime k}= \begin{cases}1, & \text { if }(i, j) \in A^{k+} \\ -1, & \text { if }(i, j) \in A^{k-}\end{cases}
$$




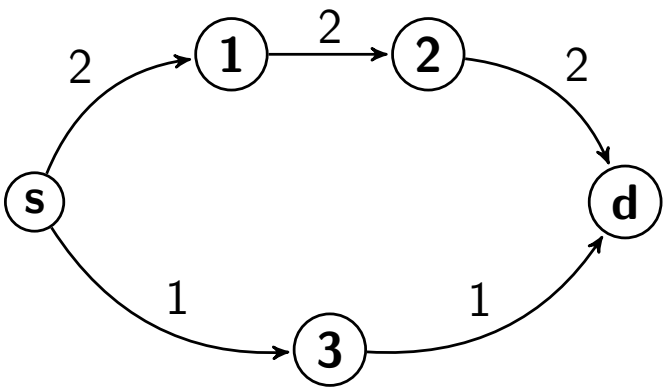

(a) Unfeasible flow.

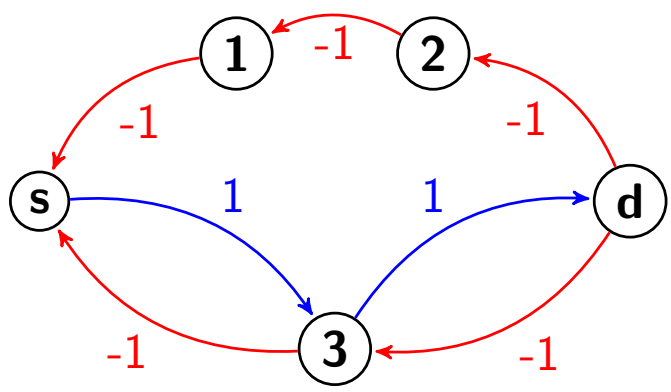

(c) The residual network of $3 a$.

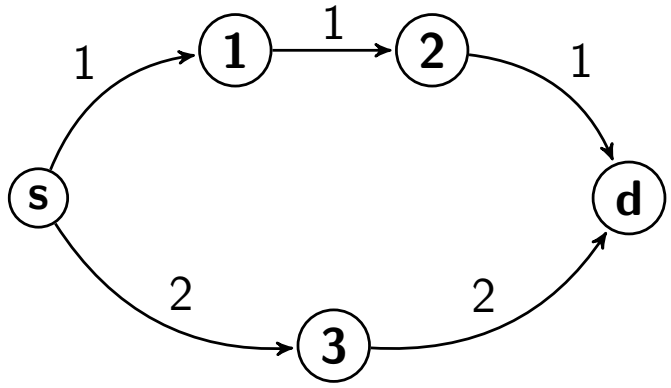

(b) Feasible flow.

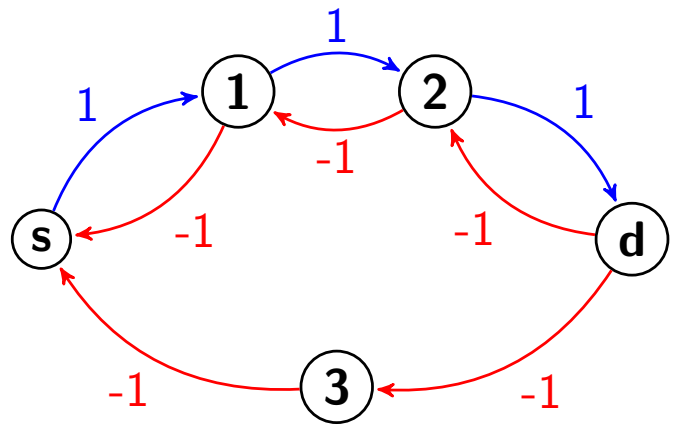

(d) The residual network of $3 \mathrm{~b}$.

Fig. 3: Example of an unfeasible (feasible) flow violating (satisfying) negative cycle conditions. All link capacities are equal to 2 with $\Phi=3$ to be sent from $s$ to $d$.

Theorem 1 (Residual network optimality ([2], chapter 9)) A feasible flow $x^{k}$ of a demand $k$ is optimal if and only if the network $G\left(x^{k}\right)$ has no negative cycles. This condition is equivalent to:

$$
c_{i j}^{\prime k}+\Pi_{i}^{k}-\Pi_{j}^{k} \geq 0, \quad \forall(i, j) \in G\left(x^{k}\right), \forall k \in K .
$$

To illustrate, let us consider the example in Figure 3. We suppose that the capacity of each link is equal to 2 and we have a demand with $\phi=3$ to be sent from the node s to d. Figure 3 represents two solutions (3a) and $(3 \mathrm{~b})$ and the associated residual networks (3c) and (3d), respectively. The solution in Figure (3a) is unfeasible because the associated residual network contains a negative cycle: s-3-d-2-1-s (resp, (3b) is feasible because (3d) contains no negative cycle).

We can model the residual network associated with a demand $k$ by using binary variables $\mu_{i j}^{k+}, \mu_{i j}^{k-}$, and the additional variables $\nu_{i j}$ for each $(i, j) \in A$. 
Proposition 1 Let $\mu \in\{0,1\}^{2 \times|A| \times|K|}, \nu \in\{0,1\}^{|A|}$ be a solution of:

$$
\begin{array}{ll}
C_{i j}-\sum_{k^{\prime} \in K} x_{i j}^{k^{\prime}} \leq C_{i j} \nu_{i j}, & \forall(i, j) \in A, \\
\mu_{i j}^{k+}+\mu_{i j}^{k-} \leq y_{e(i, j)}, & \forall(i, j) \in A, \\
\mu_{i j}^{k-} \leq x_{j i}^{k} & \forall(i, j) \in A, \\
C_{j i} \mu_{i j}^{k-} \geq x_{j i}^{k} & \forall(i, j) \in A, \\
C_{i j} \mu_{i j}^{k+} \leq C_{i j}-\sum_{k^{\prime} \in K} x_{i j}^{k^{\prime}} & \forall(i, j) \in A, \\
\mu_{i j}^{k+} \geq y_{e}-\mu_{i j}^{k-}+\nu_{i j}-1 & \forall(i, j) \in A, \\
\mu_{i j}^{k+}, \mu_{i j}^{k-} \in\{0,1\} & \forall(i, j) \in A, \\
\nu_{i j} \in\{0,1\} & \forall(i, j) \in A .
\end{array}
$$

1. For each $(i, j) \in A, \nu_{i j}=1$ if: $\sum_{k \in K} x_{i j}^{k}<C_{i j}$.

2. $\operatorname{Arc}(i, j) \in A^{k-}(x)$ iff: $\mu_{i j}^{k-}=1$.

3. $\operatorname{Arc}(i, j) \in A^{k+}(x)$ iff: $\mu_{i j}^{k+}=1$.

Proof We see that the set of constraints $6 \mathrm{a}-6 \mathrm{~h}$ ) is decomposable by arc. Hence, consider an arc $(i, j) \in A$.

1. is guaranteed by constraints $6 \mathrm{a}$.

2. is guaranteed by constraints $(6 \mathrm{c})$ and $6 \mathrm{~d})$ :

$$
\begin{array}{llll}
-(i, j) \notin A^{k-} \Rightarrow & x_{j i}^{k}=0 \stackrel{\text { (6c) }}{\Longrightarrow} & \mu_{i j}^{k-}=0 . \\
-(i, j) \in A^{k-} \Rightarrow & x_{j i}^{k}>0 \stackrel{\text { (6d) }}{\Longrightarrow} & \mu_{i j}^{k-}=1 .
\end{array}
$$

3 . is guaranteed by constraints $(6 \mathrm{a})-(6 \mathrm{f})$ :

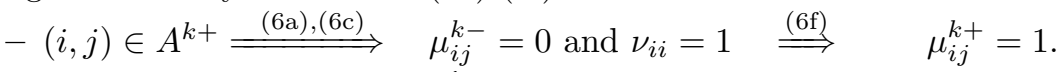

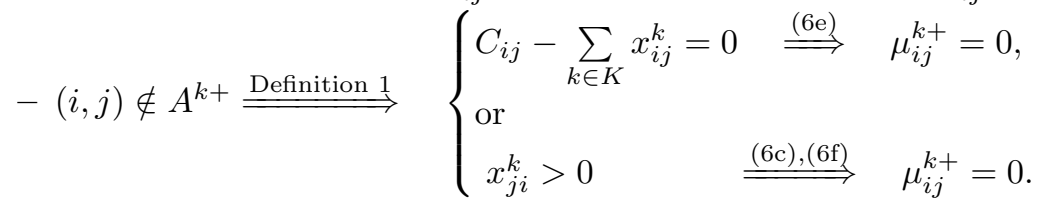

Hence, for any solution to (6), arc $(i, j) \in A^{k+}(x)$ (resp. $\left.(i, j) \in A^{k-}(x)\right)$ if $\mu_{i j}^{k+}=1$ (resp. $\left.\mu_{i j}^{k-}=1\right)$.

Using Theorem 1 and Proposition 1, the ETE-MPR problem can be reformulated with the residual network optimality conditions. 
$\min \sum_{i \in V} P_{i} z_{i}+\sum_{(i, j) \in A} \sum_{k \in K} g_{i j} x_{i j}^{k}$

s.t. $1 \mathrm{~b}-6 \mathrm{e}),(6 \mathrm{a})-6 \mathrm{bh})$,

$$
\begin{array}{lr}
\sum_{(i, j) \in \delta^{+}(i)} x_{i j}^{k}-\sum_{(i, j) \in \delta^{-}(i)} x_{j i}^{k}=b_{i}^{k}, & \forall i \in V, \forall k \in K, \\
\sum_{k \in K} x_{i j}^{k} \leq C_{i j} y_{e(i, j)}, & \forall(i, j) \in A, \\
1+\pi_{i}^{k}-\pi_{j}^{k} \geq M\left(\mu_{i j}^{k+}-1\right) & \forall(i, j) \in A, \forall k \in K \\
-1+\pi_{i}^{k}-\pi_{j}^{k} \geq M\left(\mu_{i j}^{k-}-1\right) & \forall(i, j) \in A, \forall k \in K \\
x_{i j}^{k} \geq 0, & \forall(i, j) \in A, \forall k \in K .
\end{array}
$$

Constraints $(7 \mathrm{c})$ and $(7 \mathrm{~d})$ eliminate negative cycles in the residual network associated to each flow $x^{k}$, for all $k \in K$. Since these constraints represent optimality condition (5a) associated to our problem.

\subsection{Single path routing}

Section 5 presents a comparison of the multi-path routing model with the existing single-path model. Thus, in this subsection, we describe the bi-level problem of minimizing energy and link utilization while using a single path protocol OSPF. The single-path routing problem consists of finding a single shortest path for each demand $k \in K$ from its source node $o(k)$ to the destination node $d(k)$. This problem can be formulated as:

$$
\begin{array}{lll}
\min & \sum_{i \in V} P_{i} z_{i}+\sum_{(i, j) \in A} \sum_{k \in K} g_{i j} \phi^{k} x_{i j}^{k} & \\
\text { s.t. } & (1 \mathrm{~b})-(1 \mathrm{e}), & \\
& \min \sum_{k \in K} \sum_{(i, j) \in A} x_{i j}^{k} & \\
& & \\
& \sum_{(i, j) \in \delta^{+}(i)} x_{i j}^{k}-\sum_{(i, j) \in \delta^{-}(i)} x_{j i}^{k}=b_{i}^{k}, & \forall i \in V, \forall k \in K, \\
& \sum_{k \in K} \phi^{k} x_{i j}^{k} \leq C_{i j} y_{e(i, j)}, & \forall(i, j) \in A, \\
& x_{i j}^{k} \in\{0,1\}, & \forall(i, j) \in A, \forall k \in K,
\end{array}
$$

where:

$$
b_{i}^{k}= \begin{cases}1, & \text { if } i=o(k), \\ -1, & \text { if } i=d(k), \\ 0, & \text { otherwise. }\end{cases}
$$


$x^{k} \in\{0,1\}^{|A|}$ represents here the path for demand $k$. Then, the flow in each arc $(i, j) \in A$ will be given by $\phi^{k} x_{i j}^{k}$ in this single-path routing model.

The follower problem is now an integer linear program. Thus, the KKT optimality conditions cannot be applied here. We propose to formulate the single-path routing problem as a one level BILP program with flow elimination constraints as:

$$
\begin{array}{lll}
\min & \sum_{i \in V} P_{i} z_{i}+\sum_{(i, j) \in A} \sum_{k \in K} g_{i j} \phi^{k} x_{i j}^{k} & \\
\text { s.t. } & {[\mathrm{1b}-1 \mathrm{e},} & \\
& \sum_{(i, j) \in \delta^{+}(i)} x_{i j}^{k}-\sum_{(i, j) \in \delta^{-}(i)} x_{j i}^{k}=b_{i}^{k}, & \forall i \in V, \forall k \in K, \quad \text { (9a) } \\
& \sum_{k \in K} \phi^{k} x_{i j}^{k} \leq C_{i j} y_{e(i, j)}, & \forall(i, j) \in A, \quad \text { (9b) } \\
& \sum_{k \in K} \sum_{(i, j) \in A} x_{i j}^{k} \leq C(P)+\left(|E(P)|-\sum_{e \in E(P)} y_{e}\right) M, & \forall P \in \mathcal{P}, \quad \text { (9c) } \\
& x_{i j}^{k} \in\{0,1\}, & \forall(i, j) \in A, \forall k \in K . \quad \text { (9d) }
\end{array}
$$

where, $\mathcal{P}$ is the set of all feasible solutions $P=\left(x^{*}, y^{*}, z^{*}\right)$, and $E(P)$ is the set of activated arcs on $P$ defined by $E(P)=\left\{e \in E: y^{*}=1\right\}$. In (9c), $C(P)$ represents the total cost of $P$, and $M$ is a precomputed large number that can be replaced by $\sum_{k \in K} \Phi_{k}$. The single-path routing problem is studied in [8] without capacity constraints, in this paper, the bi-level problem is reformulated using the constraint (9c) which gave good results comparing the other proposed reformulations.

\section{Algorithms}

In Subsections 3.2, 3.3, and 3.4 we presented three different formulations for ETE-MPR the problem. Formulations (3) and (7) are compact ones, while formulation (4) may have an exponential number of constraints. In this section, first, we focus on presenting different exact methods to deal with the problem formulations (Subsections 4.1, 4.2 and 4.3). Then, we present how formulation (8) will be solved (Subsection 4.4).

\subsection{Compact formulations}

The first way to solve the problem is to feed the one level models based on KKT (3) and residual network optimality conditions (7) to an ILP solver with default parameters. Let KKT and RN denote the solution methods obtained, respectively. 
4.2 Cutting plane algorithm

In this subsection, we discuss how to solve our problem with a cutting plane (CP-FC) algorithm based on the MILP formulation (4). This algorithm is described in the following:

1. Let $\mathcal{P}$ be the first level problem minimizing the sum of the energy consumption and the arc utilization:

$$
\begin{array}{ll}
\min & \sum_{i \in V} P_{i} z_{i}+\sum_{(i, j) \in A} \sum_{k \in K} g_{i j} x_{i j}^{k} \\
\text { s.t. } & 1 \mathrm{~b}-1 \mathrm{e}, 1 \mathrm{~g}-1 \mathrm{i} .
\end{array}
$$

2. Solve $\mathcal{P}$ and let $F^{*}=\left(z^{*}, y^{*}, x^{*}\right)$ be the solution obtained.

3. Let $N^{*}$ be the subnetwork defined by the set of activated routers $\left(z^{*}, y^{*}\right)$, and solve the second level problem (minimization of the link capacity utilization) in $N^{*}$. Let $x^{\prime}$ be the flow obtained and $F^{\prime}=\left(z^{*}, y^{*}, x^{\prime}\right)$.

4. If $C(F)=C\left(F^{\prime}\right)$, then the solution $\left(z^{*}, y^{*}, x^{*}\right)$ is optimal, stop. Otherwise, add the inequality (4b) associated with the solution $F^{*}$ as a new constraint to $\mathcal{P}$ in order to cut the unfeasible solution $\left(z^{*}, y^{*}, x^{*}\right)$.

5. Go to step 2. The procedure described above is repeated until the optimal solution is found.

\subsection{Branch-and-cut algorithm}

The one-level formulation (4) can also be used to solve the ETE-MPR problem by a branch-and-cut (B\&C-FC) algorithm.

The main idea of the algorithm used here is to solve the first level problem (without taking in consideration the minimization of the total traffic) by a branch-and-bound procedure and to add valid inequalities (4b) at each integral node of branch-and-bound tree violating the min-cost flow constraints. Once an integral node of the branch-and-bound tree is reached, i.e. a node where an integer solution has been found, if an inequality (4b) is violated, it is added to the problem through callbacks. In the sequence, the algorithm continues the search for an integer optimal solution satisfying all flow constraints. The algorithm stops when there are no more nodes to evaluate, i.e., all the required missing flow constraints $4 \mathrm{~b}$ were generated.

The difference between this algorithm and the iterative cutting plane algorithm presented in Subsection 4.2 lies in solving a unique MILP while one MILP is solved from scratch at each iteration of the algorithm previous presented.

\subsection{Single path routing}

The single-path routing is a generalization of the fixed charge network design problem with shortest path constraints (FCNDP-SCP) studied in [8]. Because 
of the capacity constraints added to the problem, the algorithms proposed in 8] are no longer applicable.

One of the ways to solve it is to use the iterative cutting plane algorithm presented in Subsection 4.2. The only difference is that we now solve the first level problem of (8), and we cut a path violating shortest path requirement in each iteration by adding valid inequalities $(9 \mathrm{c})$.

\section{Numerical results}

In this section, we present two different experiments. First, we compare in Subsection 5.2 the CPU time of the algorithms presented in subsections 4.1 . 4.3 to obtain optimal solutions. Then, in order to compare our multi-path bi-level model with the existing single-path model, numerical evaluations are performed to compare the improvement on values of optimal solutions in Subsection 5.3 .

All algorithms are implemented in Julia 0.6.0, and the problems are solved using Gurobi 7.5.1 with default parameters and a time limit of 3600s. Simulations were performed on an Intel(R)core TM i7-6500M CPU@2.50 2.60GHz computer with $8 \mathrm{~GB}$ of RAM.

\subsection{Instances set}

Our computational experiments have been carried out on different network topologies provided by [24] and depicted in Figure 4, Abilene (4a), Polska (4b), and Geant (4c) networks. The capacity of line cards and the energy consumption of each chassis are randomly chosen from the three cases in Table 1 (Table 1 [3]).

\begin{tabular}{l|lcc}
\hline \hline \multicolumn{3}{c}{ Chassis Features } \\
\hline Case & device & capacity & hourly power consumption \\
\hline 1 & Gigabit-Eth 1 port & $2 \mathrm{Gbps}$ & $7.3 \mathrm{~W}$ \\
2 & Fast-Eth 12 ports & $2.4 \mathrm{Gbps}$ & $18.6 \mathrm{~W}$ \\
3 & Gigabit-Eth 4 ports & $8 \mathrm{Gbps}$ & $31 \mathrm{~W}$ \\
4 & SONET /SDH OC-48c & $5 \mathrm{Gbps}$ & $41.4 \mathrm{~W}$ \\
\hline \hline
\end{tabular}

Table 1: Router chassis and cards

Five different instances are generated for each network topology. Let $\left|V_{e}\right|$ be the number of nodes selected to be origin or destination point, and $|K|$ is the number of demands. Network instances are summarized in Table 2 .

The demand between each origin-destination pair is randomly generated in $[100,700]$ Mgbs. 


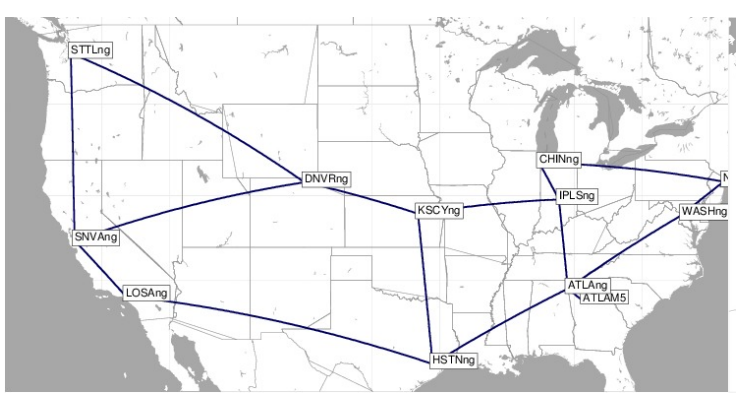

(a) Abilene network

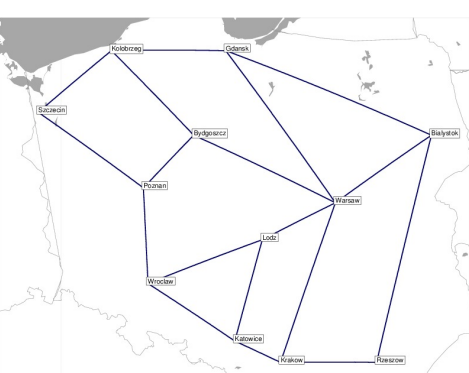

(b) Polska network

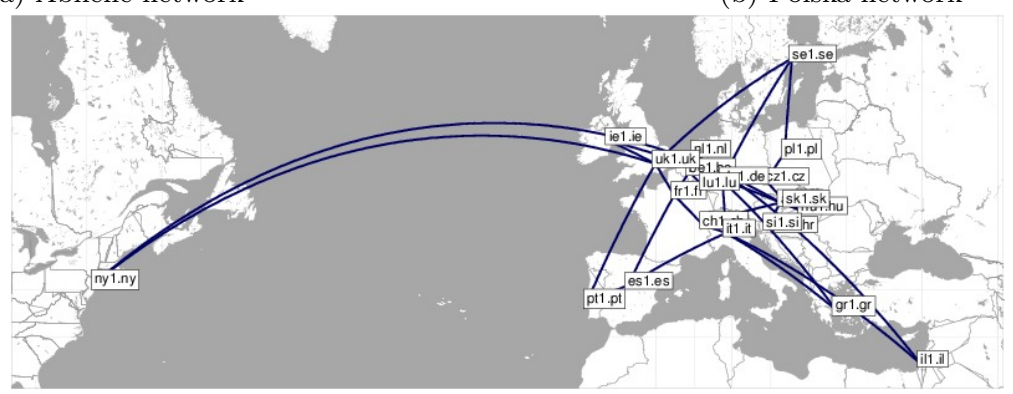

(c) Geant network

Fig. 4: Examined network topologies: (a) Abilene (b) Polska (c) Geant 24].

\begin{tabular}{|c|c|c|c|c|c|c|c|c|c|c|c|c|}
\hline \multicolumn{3}{|c|}{ Instances } & \multicolumn{2}{|c|}{1} & \multicolumn{2}{|c|}{2} & \multicolumn{2}{|c|}{3} & \multicolumn{2}{|c|}{4} & \multicolumn{2}{|c|}{5} \\
\hline Network & ||$V \mid$ & $|E|$ & $\left|V_{e}\right|$ & $|K|$ & $\left|V_{e}\right|$ & $|K|$ & $\mid V_{e}$ & $|K|$ & $\left|V_{e}\right|$ & $\mid \overline{|K|}$ & $\mid V_{e}$ & $\mid \overline{|K|}$ \\
\hline Abilene & 12 & 15 & 4 & 5 & 5 & 10 & 6 & 20 & 7 & 30 & 8 & 40 \\
\hline Polska & 12 & 18 & 4 & 5 & 5 & 10 & 6 & 20 & 7 & 30 & 8 & 40 \\
\hline Geant & 22 & 36 & 4 & 5 & 5 & 10 & 6 & 20 & 7 & 30 & 8 & 40 \\
\hline
\end{tabular}

Table 2: Network topologies used.

5.2 Solution times

\begin{tabular}{ccccc}
\hline \hline Instances & KKT & RN & CP-FC & B\&C-FC \\
\hline 1 & 0.08 & 0.08 & 0.03 & 0.34 \\
2 & 0.35 & 0.94 & 0.09 & 0.06 \\
3 & 2.31 & 3.47 & 0.10 & 0.07 \\
4 & 6.84 & 6.11 & 0.10 & 0.11 \\
5 & 19.19 & 10.12 & 0.12 & 0.11 \\
\hline Average & 5.76 & 4.14 & 0.09 & 0.14 \\
\hline \hline
\end{tabular}

Table 3: Comparison of CPU time on Abilene network. 
First, we compare the different algorithms presented in subsections 4.14 .3 with respect to solution times. The obtained results are reported in Tables 3. 4 and 5 These tables present for each instance, the CPU time in seconds spent by the different algorithms to get the optimal solutions. The symbol in the tables of results "-" means that the algorithm was not able to find the optimal solution in the time limit of $3600 s$ for the considered instance.

\begin{tabular}{ccccc}
\hline \hline Instances & KKT & RN & CP-FC & B\&C-FC \\
\hline 1 & 0.56 & 0.35 & 0.31 & 1.37 \\
2 & 4.63 & 0.53 & 0.28 & 0.40 \\
3 & 3600.02 & 4.03 & 0.82 & 0.67 \\
4 & - & 185.96 & 4.67 & 2.08 \\
5 & - & 3180.68 & 86.03 & 1.01 \\
\hline Average & 1201.74 & 674.31 & 18.42 & 1.11 \\
\hline \hline
\end{tabular}

Table 4: Comparison of CPU time on Geant network.

The results show that the iterative cutting plane and branch-and-cut algorithms are close in terms of $\mathrm{CPU}$ time and outperform significantly the compact formulations. Also, We can remark, comparing the different tables, that the Geant instance (Figure (4c)) is more difficult than the other instances in terms of CPU time. If we consider, for instance, the cutting plane algorithm, the CPU time average to find the optimal solutions for Geant instance is more than 200 times the CPU time of Abilene network and 100 times the CPU time of Polska network. Furthermore, for all instances, we observe that the first scenarios 1,2, and 3 are easier than the last ones 4 and 5 according to the running time of all algorithms. These results can be explained by the difference in the size and the number of demands in each scenario and instance.

Furthermore, the residual network formulation is faster than the formulation based on KKT conditions.

\subsection{Single path routing vs multi-path routing}

We compared our multi-path model with the single-path approach (where each demand uses one path).

\begin{tabular}{ccccc}
\hline \hline Instances & KKT & RN & CP-FC & B\&C-FC \\
\hline 1 & 0.17 & 0.16 & 0.04 & 0.23 \\
2 & 1.18 & 1.80 & 0.21 & 0.16 \\
3 & 0.67 & 2.33 & 0.22 & 0.12 \\
4 & 11.83 & 24.26 & 0.22 & 0.18 \\
5 & 1453.72 & 7.38 & 0.21 & 0.11 \\
\hline Average & 293.51 & 7.18 & 0.18 & 0.16 \\
\hline \hline
\end{tabular}

Table 5: Comparison of CPU time on Polska network. 


\begin{tabular}{|c|c|c|c|c|c|c|}
\hline & \multicolumn{2}{|c|}{ Abilene network } & \multicolumn{2}{|c|}{ Geant network } & \multicolumn{2}{|c|}{ Polska network } \\
\hline Instances & multi-path & single path & multi-path & single path & multi-path & single path \\
\hline 1 & 21531.7 & 21531.7 & 20856.8 & 20856.8 & 33900.4 & 33900.4 \\
\hline 2 & 97076.9 & 97076.9 & 35949.8 & 35949.8 & 67535.8 & 67535.8 \\
\hline 3 & 199014.3 & 199512.3 & 118786.9 & 132426.9 & 104429.8 & 107261.8 \\
\hline 4 & 200820.3 & 204881.3 & 220255.3 & 223851.4 & 176674.7 & 181298.7 \\
\hline 5 & 220454.3 & 224991.3 & 200819.3 & 203373.3 & 290679.3 & 291752.3 \\
\hline Average & 147779.5 & 149598.7 & 119333.62 & 123291.64 & 134644 & 136349.8 \\
\hline
\end{tabular}

Table 6: Comparison of the first level solution of the multi-path and the singlepath approaches.

\begin{tabular}{c|cc|cc|cc}
\hline \hline & \multicolumn{2}{|c|}{ Abilene network } & \multicolumn{2}{c|}{ Geant network } & \multicolumn{2}{c}{ Polska network } \\
\hline Instances & multi-path & single path & multi-path & single path & multi-path & single path \\
1 & 4908 & 4908 & 6423 & 6423 & 7848 & 7848 \\
2 & 21791 & 21791 & 13018 & 13018 & 14410 & 14410 \\
3 & 39572 & 39771 & 31941 & 32396 & 26346 & 26346 \\
4 & 40642 & 42176 & 49948 & 51548 & 36688 & 36688 \\
5 & 44003 & 45892 & 50105 & 50450 & 56448 & 56459 \\
\hline Average & 30183.2 & 30907.6 & 30287 & 30767 & 28348 & 28350.2 \\
\hline \hline
\end{tabular}

Table 7: Comparison of the second level solution of the multi-path and the single-path approaches.

Tables 6 and 7 summarize the obtained results. For each instance, we compare the first and the second level optimal solution obtained under the two settings: single and multi-path routing. We can easily remark that using the multi-path leads to lower network energy consumption (the first level objective function) for $9 / 15$ instances. This is because in the single-path approach, we need to activate more routers and links in the case of insufficient link capacity, unlike the multi-path where the flow can be distributed. In addition, and for the same reason, in $7 / 15$ instances, the total traffic (the second level problem) is reduced. To conclude, Table 8 reports the percentage of the reduction of energy consumption (First level objective) and capacity link utilization (Second level objective) when we use the multi-path approach. It is clear that, in the worst case, the two models give the same solution. Lastly, the quantity of the energy and the capacity of links saved even if the average the obtained gaps are small. Considering for example, the third instance of Geant network, the percentage of the energy reduced is small $(10.30 \%)$, but, it represents more than 13000 (unity of energy).

\section{Conclusion}

In this paper, we have studied the problem of Energy-aware Traffic Engineering using a multi-path routing protocol (ETE-MPR) to minimize link capacity utilization in ISP backbone networks. To formulate the problem, we proposed a new bi-level optimization model where the objective of the upper-level problem 


\begin{tabular}{c|cc|cc|cc}
\hline \hline & \multicolumn{2}{|c|}{ Abilene network } & \multicolumn{2}{c|}{ Geant } & network & \multicolumn{2}{c}{ Polska network } \\
\hline Instances & First obj & second obj & First obj & second obj & First obj & second obj \\
1 & 0 & 0 & 0 & 0 & 0 & 0 \\
2 & 0 & 0 & 0 & 0 & 0 & 0 \\
3 & 0.24 & 0.50 & 10.30 & 1.40 & 2.64 & 0 \\
4 & 1.98 & 3.64 & 1.60 & 3.10 & 2.55 & 0 \\
5 & 2.02 & 4.12 & 1.26 & 0.68 & 0.37 & 0.02 \\
\hline Average & 0.85 & 1.65 & 2.63 & 1.04 & 1.11 & 0.004 \\
\hline \hline
\end{tabular}

Table 8: Percentage of the reduction on the first and the second level objectives using the multi-path model.

is to activate network devices minimizing the energy consumption, and the lower level represents a multi-path routing protocol. Then, we presented two one-level MILP formulations, respectively, based on complementarity slackness optimality and on residual network conditions and a BILP formulation using unfeasible flow elimination constraint.

The obtained results show that the iterative cutting plane and branchand-cut algorithms are close in terms of CPU time and outperform the compact formulations. Furthermore, the residual network formulation obtained the optimal solution in CPU time faster than the formulation based on KKT conditions.

\section{References}

1. Addis, B., Capone, A., Carello, G., Gianoli, L.G., Sansò, B.: Energy management through optimized routing and device powering for greener communication networks. IEEE/ACM Trans. Netw. 22(1), 313-325 (2014)

2. Ahuja, R.K., Magnanti, T.L., Orlin, J.B.: Network Flows: Theory, Algorithms, and Applications. Prentice-Hall, Inc., Upper Saddle River, NJ, USA (1993)

3. Amaldi, E., Capone, A., Coniglio, S., Gianoli, L.G.: Energy-aware traffic engineering with elastic demands and MMF bandwidth allocation. In: Proc. of IEEE CAMAD'13, pp. 169-174 (2013)

4. Andrae, A.S.G., Edler, T.: On global electricity usage of communication technology: Trends to 2030. Challenges 6(1), 117-157 (2015)

5. Banner, R., Orda, A.: Multipath routing algorithms for congestion minimization. IEEE/ACM Trans. Netw. 15(2), 413-424 (2007)

6. Bazaraa, M.S., Jarvis, J.J., Sherali, H.D.: Linear programming and network flows. John Wiley \& Sons (2011)

7. Bolla, R., Bruschi, R., Davoli, F., Cucchietti, F.: Energy efficiency in the future internet: A survey of existing approaches and trends in energy-aware fixed network infrastructures. IEEE Commun. Surveys Tuts. 13(2), 223-244 (2011)

8. Bouras, I., Figueiredo, R., Poss, M., Zhou, F.: On two new formulations for the fixed charge network design problem with shortest path constraints. Computers \& Operations Research 108, 226-237 (2019)

9. Chen, X., Jukan, A., Drummond, A.C., da Fonseca, N.L.S.: A multipath routing mechanism in optical networks with extremely high bandwidth requests. In: Proc. of IEEE Globecom'09, pp. 1-6 (2009)

10. Chiaraviglio, L., Mellia, M., Neri, F.: Minimizing isp network energy cost: Formulation and solutions. IEEE/ACM Trans. Netw. 20(2), 463-476 (2012)

11. Chiesa, M., Kindler, G., Schapira, M.: Traffic engineering with equal-cost-multipath: An algorithmic perspective. IEEE/ACM Trans. Netw. 25(2), 779-792 (2017) 
12. Cianfrani, A., Eramo, V., Listanti, M., Polverini, M., Vasilakos, A.V.: An OSPFIntegrated Routing Strategy for QoS-Aware Energy Saving in IP Backbone Networks. IEEE Trans. Netw. Service Manag. 9(3), 254-267 (2012)

13. CISCO: CISCO Visual Networking Index. Tech. rep. (2019). https://www.cisco.com/c/en/us/solutions/collateral/service-provider/ visual-networking-index-vni/white-paper-c11-738429.html\#_Toc953325

14. Dabaghi, F., Movahedi, Z., Langar, R.: A survey on green routing protocols using sleepscheduling in wired networks. J. Netw. Comput. Appl. 77(C), 106-122 (2017)

15. Erkut, E., Gzara, F.: Solving the hazmat transport network design problem. Computers \& Operations Research 35(7), 2234-2247 (2008)

16. Gupta, M., Singh, S.: Greening of the internet. In: Proc. of ACM SIGCOMM'03, pp. 19-26 (2003)

17. He, J., Song, W.: Achieving near-optimal traffic engineering in hybrid software defined networks. In: Proc. of IFIP Networking'05, pp. 1-9 (2015)

18. Lambert, S., Heddeghem, W.V., Vereecken, W., Lannoo, B., Colle, D., Pickavet, M.: Worldwide electricity consumption of communication networks. Opt. Express 20(26), B513-B524 (2012)

19. Lee, G.M., Choi, J.S.: A survey of multipath routing for traffic engineering. Information and Communications University pp. 1-27 (2018)

20. Li, M., Lukyanenko, A., Ou, Z., Ylä-Jääski, A., Tarkoma, S., Coudron, M., Secci, S.: Multipath transmission for the internet: A survey. IEEE Commun. Surveys Tuts. 18(4), 2887-2925 (2016)

21. Liu, X., Mohanraj, S., Pioro, M., Medhi, D.: Multipath routing from a traffic engineering perspective: How beneficial is it? In: Proc. of IEEE ICNP'14, pp. 143-154 (2014)

22. Mauttone, A., Labbé, M., Figueiredo, R.: A tabu search approach to solve a network design problem with user-optimal flows. In: ALIO/EURO Workshop on Applied Combinatorial Optimization (2007)

23. Merindol, P., Pansiot, J.J., Cateloin, S.: Improving load balancing with multipath routing. In: Proc. of IEEE ICCCN'08, pp. 1-8 (2008)

24. Orlowski, S., Pióro, M., Tomaszewski, A., Wessäly, R.: SNDlib 1.0-Survivable Network Design Library. In: Proceedings of the 3rd International Network Optimization Conference (INOC 2007), Spa, Belgium (2007). URL http://www.zib.de/orlowski/Paper/ OrlowskiPioroTomaszewskiWessaely2007-SNDlib-INOC.pdf.gz Http://sndlib.zib.de, extended version accepted in Networks, 2009.

25. Singh, S.K., Das, T., Jukan, A.: A survey on internet multipath routing and provisioning. IEEE Commun. Surveys Tuts. 17(4), 2157-2175 (2015)

26. Zhang, J., Xi, K., Chao, H.J.: Load balancing in ip networks using generalized destination-based multipath routing. IEEE/ACM Trans. Netw. 23(6), 1959-1969 (2015) 